\title{
Analysis of Fluid-thermal Coupling in Ferrofluid Bearing Used in High Speed Machines
}

\author{
Xin Yin *, Jien Ma *, Youtong Fang *, and Shuai Jin *
}

\begin{abstract}
This paper describes analysis of journal bearings lubricated with ferrofluid, which are very suitable for high speed machines. Comparing to conventional lubricants, the coupling of hydrodynamic, thermal and magnetic properties of ferrofluid adds to the complexity in analysis. Modified Reynolds equation and energy equation are derived and solved numerically using finite volume method. Pressure distribution is got which takes temperature effect into consideration. Static characteristics are then discussed. One optimal scheme is also got according to analysis results.
\end{abstract}

Keywords: Ferrofluid bearing, Finite volume method, Hydrodynamic, Numerical, Thermal

\section{Introduction}

Ferrofluid is a kind of colloidal liquid consisting of nano-scale ferromagnetic particles suspended in carrier fluid (Fig. 1). The particles are coated by surfactant, Van der Waals force of which helps to prevent clumping [1]. Like other ferromagnetic materials, ferrofluids will be strongly magnetized in the presence of external magnetic field. When the external field is cancelled, magnetization disappears. Ferrofluids also have characteristics of any other fluids [2]. According to above features, ferrofluids can be used in varies areas for different purposes such as sealing, lubricating and heat transfer [3]. Ferrofluid bearing (Fig. 2), or hydro-magnetic bearing, which replaces traditional lubricant with ferrofluid, was developed in 1960s in order to coordinate with nuclear device. Lubricating with ferrofluid has several advantages [4]: better lubricating characteristic, less leakage, controllable viscosity, etc.

Lots of work has been done about ferrofluid since 1940s when it was first invented. Tipei N. has derived a pressure differential equation which is equivalent Reynolds equation and further studied several characteristics about ferrofluid bearing in [5]. From which he concludes that ferromagnetic lubricants may improve the performance of bearings under low loads and at low speed. He assumed an axial parabolic magnetic field distribution, which is also used by H. S. Chang in [6]. T. A. Osman derived modified Reynolds equation and use finite differential method solving it numerically to study the static characteristics of magnetized jour-

* College of Electrical Engineering, Zhejiang University, Hangzhou, China. (yx123156@gmail.com, majien@zju.edu.cn, youtong @ zju.edu.cn,21010058@zju.edu.cn)

Received 10 November 2013; Accepted 20 November 2013 nal bearings [7]. He also uses dynamic coefficients as input data to study the stability of the rotor bearing system. All of them neglect thermal influence. H. Yang uses commercial

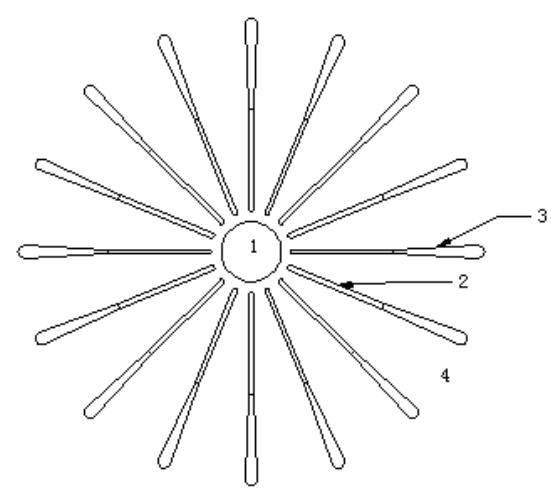

1: Nano-scale magnetic particle 2: Inner layer of surfactant 3: Outer layer of surfactant

Fig. 1. Magnetic particle coated with surfactant in carrier fluid.

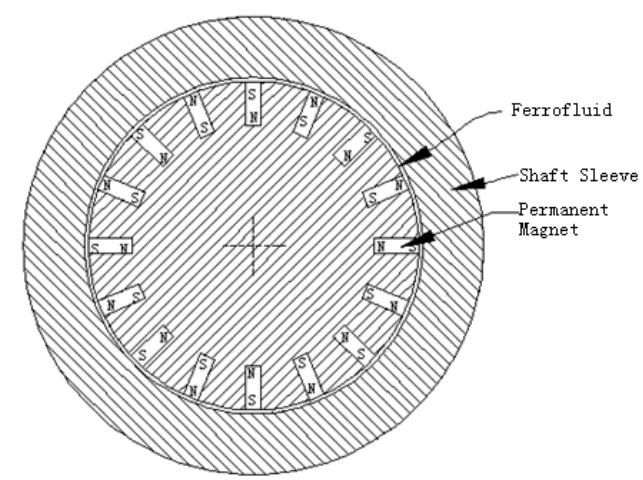

Fig.2. One possible structure of ferrofluid bearings 
FEM (finite element method) software (ANSYS) to simulate the characteristics of ferrofluid bearing in 2D [8]. S. Jin extends the conclusion into 3D and explores the effect of eccentricity. However, the model used is somehow coarse and a lot of factors are out of consideration [9]. In this paper, we follow T. A. Osman's study but introduce thermal analysis into solving process. Finite volume method is also used instead of FDM.

\section{Theoretical Analysis}

\subsection{D Modified Reynolds Equation}

The cross section of bearing is as Fig. 3. Eccentricity and break of the film are considered in this model.

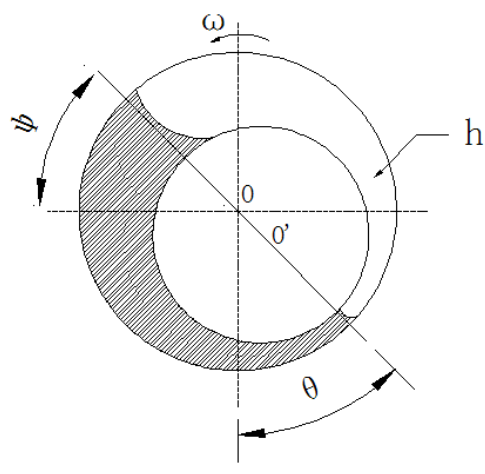

Fig. 3. Cross-section view of bearing

The shaft is rotating counterclockwise at angular velocity $\omega$. Angle counterclockwise from thickest point is denoted by $\Psi$ and clockwise from the thinnest point is $\theta$. Film thickness is $\mathrm{h}$ and in non-dimension form it can be expressed as:

$$
H=1+\varepsilon \cdot \mathrm{co} \mathrm{o} \phi
$$

From T.A. Osman's study, we can derive modified Reynolds equation for ferrofluid film. Coordinate system here is defined as below: $\mathrm{x}, \mathrm{y}$ and $\mathrm{z}$ represent the circumferential, radical and axial direction of the bearing, respectively. In existence of external magnetic field, induced magnetic force in unit volume can be expressed as:

$$
\boldsymbol{F}_{\boldsymbol{m}}=\left(\nabla \times \boldsymbol{h}_{\boldsymbol{m}}\right) \times \boldsymbol{B}+\mu_{0} \boldsymbol{M}_{g}\left(\nabla \boldsymbol{h}_{\boldsymbol{m}}\right)
$$

Because of the low conductivity of carrier fluid, ferrofluid can be trusted as non-conductor, thus no axial current exists and the curl term of magnetic intensity can be omitted. Finally, magnetic force is:

$$
\boldsymbol{F}_{\boldsymbol{m}}=\mu_{0} \boldsymbol{M}_{\boldsymbol{g}}\left(\nabla \boldsymbol{h}_{\boldsymbol{m}}\right)=\mu_{0} X_{m} \boldsymbol{h}_{\boldsymbol{m}}\left(\nabla \boldsymbol{h}_{\boldsymbol{m}}\right)
$$

From basic Navier-Stokes equation, considering magnetic force as a body force, the motion equation for ferrofluid film can be derived:

$$
\left\{\begin{array}{l}
\eta \frac{\partial^{2} u}{\partial y^{2}}=\frac{\partial p}{\partial x}-f_{m x} \\
\eta \frac{\partial^{2} u}{\partial y^{2}}=\frac{\partial p}{\partial z}-f_{m z}
\end{array}\right.
$$

Film thickness is small enough that it's reasonable to assume there's no variation of pressure in radical direction, that is to say, $p$ is not a function of $y$. Integrating (2) twice with boundary condition $u(h)=\omega r, v(h)=u(0)=v(0)=0$ expressions for circumferential and axial velocity are:

$$
\left\{\begin{array}{l}
u=\frac{1}{2 \eta} \frac{\partial p}{\partial x}\left(y^{2}-y h\right)-\frac{1}{2 \eta} f_{m x}\left(y^{2}-y h\right)+\omega r \frac{y}{h} \\
v=\frac{1}{2 \eta} \frac{\partial p}{\partial z}\left(y^{2}-y h\right)-\frac{1}{2 \eta} f_{m z}\left(y^{2}-y h\right)
\end{array}\right.
$$

In this paper, magnetic field distribution is assumed to be axial parabolic [3]. Magnetic components are got:

$$
h_{m}(z)=h_{m c}-\left(h_{m c}-h_{m e}\right)(2 z / L)^{2}
$$

Under this assumption, magnetic force only exists in axial direction and $f_{m x}$ is zero. Combining the continuity equation, finally the modified Reynolds equation is:

$$
\frac{\partial}{\partial x}\left(h^{3} \frac{\partial p}{\partial x}\right)+\frac{\partial}{\partial z}\left(h^{3} \frac{\partial p}{\partial z}\right)=6 \eta \omega r \frac{\partial h}{\partial x}+\frac{\partial}{\partial z}\left(h^{3} f_{m z}\right)
$$

To simplify numerical solving process, a dimensionless form is used actually:

$$
\begin{aligned}
& \frac{\partial}{\partial \theta}\left(H^{3} \frac{\partial P}{\partial \theta}\right)+\frac{1}{4 v^{2}} \frac{\partial}{\partial \lambda}\left(H^{3} \frac{\partial P}{\partial \lambda}\right)= \\
& \frac{6 \eta \omega r}{c^{2} \cdot p_{0}}+\frac{\mu_{0} X_{m} c^{2} h_{0}^{2}}{\eta \omega l^{2}} \frac{\partial}{\partial \lambda}\left(H^{3} H_{m} \frac{\partial H_{m}}{\partial \lambda}\right)
\end{aligned}
$$

By substituting as following equations:

$$
\left\{\begin{array}{l}
p_{0}=\frac{\eta \omega r^{2}}{l^{2}} \\
\alpha=\frac{\mu_{0} X_{m} h_{m 0}{ }^{2}\left(h_{0}{ }^{2}\right)}{\eta \omega l^{2}}
\end{array}\right.
$$


The final equation is:

$$
\begin{aligned}
& \frac{\partial}{\partial \theta}\left(H^{3} \frac{\partial P}{\partial \theta}\right)+ \frac{1}{4 v^{2}} \frac{\partial}{\partial \lambda}\left(H^{3} \frac{\partial P}{\partial \lambda}\right)= \\
& 6 \frac{\partial H}{\partial \theta}+\alpha \frac{\partial}{\partial \lambda}\left(H^{3} H_{m} \frac{\partial H_{m}}{\partial \lambda}\right)
\end{aligned}
$$

Boundary condition is needed to guarantee unique solution [10]. Reynolds B.C. is most used which thinks that film is not continuous and breaks at angle $\varphi 1$. Under Reynolds condition, pressure of cave region is forced to be zero. It can be described as:

$$
\left\{\begin{array}{l}
p(\varphi)=\frac{\partial p}{\partial \varphi}=0, \varphi=\varphi_{1} \\
p(\varphi)=0, \quad \varphi_{1}<\varphi<2 \pi
\end{array}\right.
$$

\subsection{Energy Equation}

Osman has studied characteristics of ferrofuid lubricant without regard to temperature variation. As a result, viscosity of ferrofluids is not exact. Since fluid viscosity influences the load carrying capacity notably, thermal analysis is taken into consideration by solving energy equation. From energy conservation law, equation is obtained:

$$
\rho c \cdot \operatorname{div}\left(u_{j} T\right)=\operatorname{div}(\Gamma \operatorname{grad} T)+S
$$

From left to right, the three terms are respectively convection, conduction and internal energy source terms. For 2D problem, convection term can be expressed as:

$$
\rho c\left(\frac{\partial T}{\partial t}+u \frac{\partial T}{\partial x}+v \frac{\partial T}{\partial y}\right)
$$

For static analysis, the first term above is equal to zero. Parameter $\Gamma$ in conduction term here represents heat conductivity coefficient $\mathrm{k}$. In 2D analysis, conduction term is:

$$
k\left(\frac{\partial^{2} T}{\partial x^{2}}+\frac{\partial^{2} T}{\partial z^{2}}\right)
$$

Since the radical velocity gradient is much greater than ones of the other two directions, the internal energy term $\mathrm{S}$ can be approximately expressed as:

$$
S=\eta\left[\left(\frac{\partial u}{\partial y}\right)^{2}+\left(\frac{\partial v}{\partial y}\right)^{2}\right]
$$

Combining convection and conduction terms into total heat flux terms, that are $J_{x}$ and $J_{y}$, simplified energy equations are:

$$
\left\{\begin{array}{l}
\frac{\partial J_{x}}{\partial x}+\frac{\partial J_{z}}{\partial z}=S \\
J_{x}=\rho u T-\frac{k}{c} \frac{\partial T}{\partial x} \\
J_{z}=\rho v T-\frac{k}{c} \frac{\partial T}{\partial z}
\end{array}\right.
$$

Similarly, boundary condition is necessary to keep solution unique. In this study, two B.C. should be taken into consideration. Firstly, bearing ends are assumed heat insulation. Reason for this is that heat conduction effect of ferrofluid is very small. Secondly, the starting point where lubricant comes into clearance is set a fixed temperature of $300 \mathrm{~K}$. Both assumptions above agree with real working condition.

\subsection{Discretization Equations}

Modified Reynolds equation and energy equation need to be solving simultaneously in order to get static characteristics of ferrofluid bearing, which is too complicated by hand. Numerical method is a good way to solve difficult part difference equation. There are several different methods to choose, such as finite differential method, finite element method, boundary element method, etc. Both two fundamental equations described above have many variables. In this case, finite volume method (FVM) is preferred. By meshing solving area into small volumes and investigating each volume, solution of the whole area is obtained. In this sight, the essential idea of FVM is similar to other numerical method, but FVM has its own characteristics and advantages. It can be regarded as a medial between FDM and FEM. FDM only cares about node solution without appointing interpolating function between nodes, which is needed in FEM. Interpolating function, is also necessary in FVM, but it will be only used to get discrete form of differential equation, which means it is useless once difference equation is obtained.

Control volume integrating is the main approach to get difference equation in FVM. Firstly, mesh solving area into small volumes. One volume is as Fig. 4. Then interpolating function should be defined.

For convenience, stepwise distribution is used here. By integrating (10) and (16) within control volume, difference form of the problem is obtained. Similarly, difference energy equation can be obtained: 


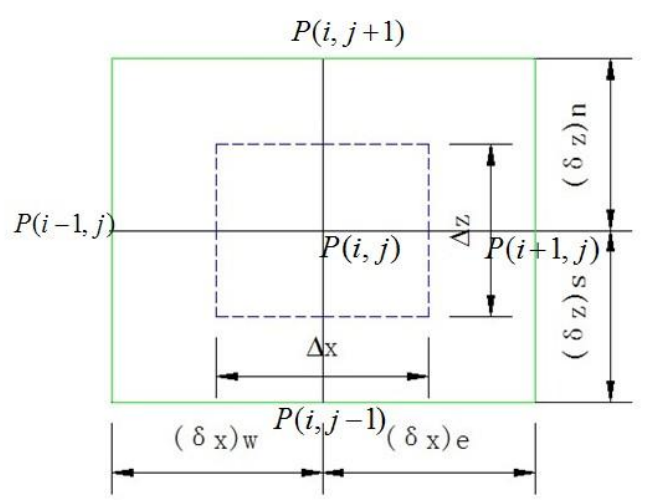

Fig. 4. Finite volume unite

$$
\beta_{P} T_{P}=\beta_{E} T_{E}+\beta_{W} T_{W}+\beta_{N} T_{N}+\beta_{S} T_{S}+\beta_{0}
$$

If handled exactly the same as modified Reynolds equation, coefficients $(\beta)$ might be negative when convection is larger than conduction, which means $a_{p}<\sum\left|a_{n b}\right|$. It fails to satisfy the Scarborough criterion and will cause divergence. So this method can only be applied under low Reynolds number. To expend this limit, upwind scheme is used.

By now, we've derived discrete form of modified Reynolds equation and energy equation note that upwind scheme is used when deriving energy equation. Because velocity used above is on the nodes. Staggered grid is used to keep solution stable.

Successive Over-Relaxation (SOR) iterative method is used rather than Gauss-Seidel method to accelerate convergence. It's a common iterative method which is widely used in numerical analysis. Take pressure for example, SOR method can be described as follow:

$$
\begin{aligned}
P_{i, j}{ }^{(k+1)}=\sigma & \left(A P_{i+1, j}+B P_{i, j+1}+C P_{i, j-1}\right. \\
& \left.+D P_{i-1, j}+E-P_{i, j}{ }^{(k)}+P_{i, j}{ }^{(k)}\right)
\end{aligned}
$$

Where $\sigma$ is iterative factor, Gauss-Seidel can be regarded as a special case if the factor is zero. Range for $\sigma$ in SOR is $1<\sigma<2$.

\subsection{Coupling Analysis}

Next problem is how to combine the two discretion equation to realize coupling analysis. The main effect of temperature and pressure is change in fluid viscosity. Like other liquid, Ferrofluid viscosity rises with increasing pressure and decreasing temperature. There are many researchers who study the law of the change in liquid viscosity, including Reynolds viscosity-temperature equation, AndradeErying viscosity-temperature equation and Vogel viscosity- temperature equation. Among these, Reynolds is the most convenient while Vogel is the most accurate.

In this paper, Reynolds viscosity-temperature equation is used:

$$
\eta=\eta_{0} \exp \left[\left(\ln \eta_{0}+9.67\right)\left(1+5.1 \times 10^{-9} p\right)^{0.68} \frac{T-138}{T_{0}-138}-1\right]
$$

Knowing the changing rules of viscosity with pressure and temperature, a bridge can be built between hydrodynamic analysis and thermal analysis.

\subsection{Static Characteristics}

If pressure distribution is obtained, it's easy to get the load carrying capacity of the film by:

$$
\left\{\begin{array}{l}
W=\sqrt{W_{\varepsilon}^{2}+W_{\phi}^{2}} \\
W_{\varepsilon}=\int_{-0.5}^{0.5} \int_{0}^{2 \pi} P \cos \varphi \cdot d \varphi \cdot d \lambda \\
W_{\phi}=\int_{-0.5}^{0.5} \int_{0}^{2 \pi} P \sin \varphi \cdot d \varphi \cdot d \lambda
\end{array}\right.
$$

Where, $W_{Z}$ and $W_{\Phi}$ are axial and circumferential component of dimensionless carrying capacity, respectively. $W$ is the total carrying capacity of ferrofluid film. To get the actual value, the basic value should be defined:

$$
\left\{\begin{array}{l}
w_{0}=\frac{\eta \omega l r^{3}}{h_{0}{ }^{2}} \\
w=W \cdot w_{0}
\end{array}\right.
$$

\section{Results and analysis}

Based on theories described above and using MATLAB to realize, the pressure distribution of lubricant film for ferrofluid bearing is as Fig. 5, from which we can see peak value locates between $\pi / 2$ and $\pi$. In cave region, pressure equals zero. By changing magnetic factor $\alpha$, different distribution of pressure are obtained in Fig. 6. As magnetic effect increased, full-lubricating region extends, which means shrinking of cave region and improving in lubricating characteristics.

Under condition that bearing clearance is $0.002 \mathrm{~m}$, rotating speed is $6000 \mathrm{r} / \mathrm{min}(628.3 \mathrm{rad} / \mathrm{s})$ and eccentricity is 0.9 , 
temperature distribution of the film is as Fig. 7. The max temperature rise is about $7 \mathrm{~K}$, which is quite small. However, since viscosity varies remarkably with temperature, this effect is worth consideration. From Fig.8, the minimum of viscosity reaches 0.85 relative to origin value, i.e. $15 \%$ drop. As a result of viscosity drop, pressure decreases accordingly. The results fit well with our theoretical analysis. When considering high speed and heavy load, temperature and pressure to fluid viscosity can't be ignored, increase in temperature causes decrease in viscosity. In contract, pressure rise brings viscosity drop. The former effect is dominant. By now we have a general concept about pressure distribution and its influence factors of ferrofluid bearing.

From data above we can further get a series of static characteristics such as load carrying capacity which can be obtained from (6-1) (6-2). In Fig. 9, we get load capacity under different magnetic intensity. Viscosity has logarithmic relationship with magnetic intensity and approximately proportion to general load capacity. Temperature rise caused by increasing viscosity weakens the rise in viscosity. On the other hand, magnetic field also applied additional force to increase load capacity. Assuming magnetic intensity $\mathrm{H}$ ranges from 1000 to $50000 \mathrm{~A} / \mathrm{m}$, Fig. 9 is obtained. Under different magnetic intensity with fixed eccentricity 0.9 , load carrying capacity rises from 4 to $13 \mathrm{kN}$. Obviously,

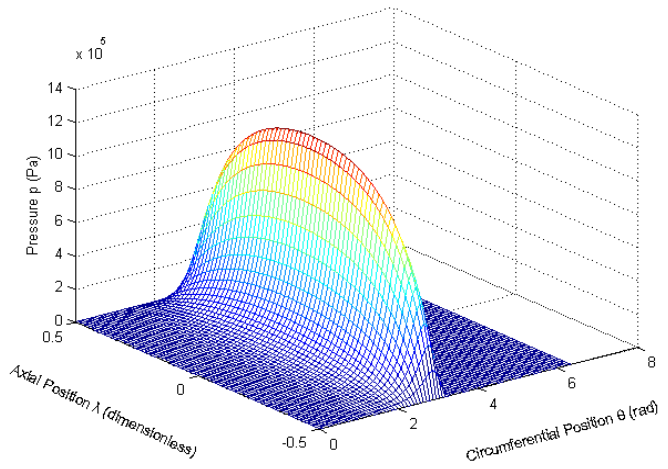

Fig. 5. Pressure distribution in lubricant region under condition that $\varepsilon=0.9, \mathrm{f}=6000 \mathrm{r} / \mathrm{min}$
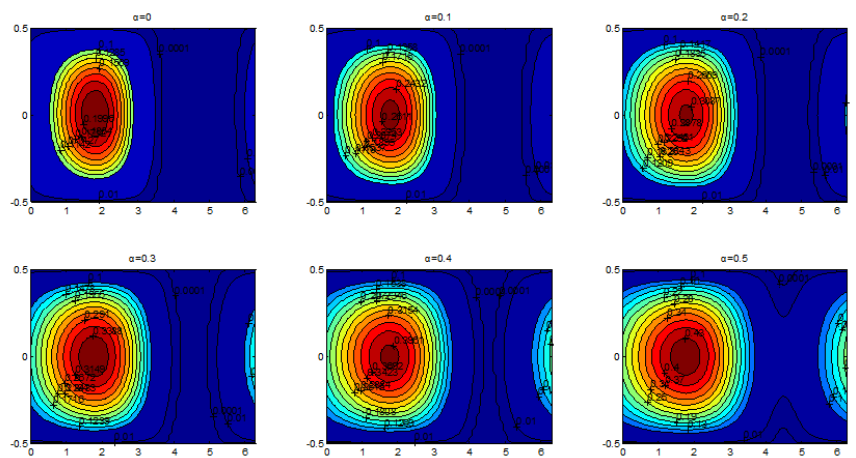

Fig. 6. Magnetic effect to lubricating region load carrying capacity is controllable by changing magnetic parameter. Exact corresponding relationship between magnetic parameter and load carrying capacity can be recorded through experiments, thus it's very convenient in practical applications. Comparing calculated value and fitted curve, a small difference is because temperature rise decreases the viscosity which is mentioned above. The stronger the magnetic field is, the smaller the difference will become. In general, load carrying capacity is increased.

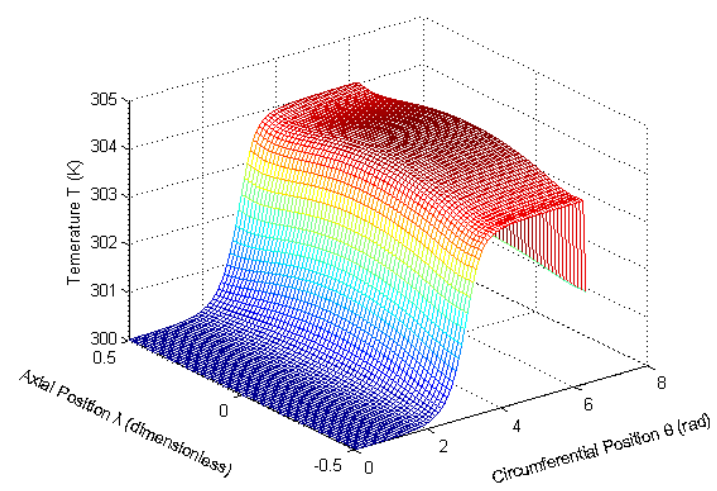

Fig. 7. Temperature distribution $\left(\mathrm{h}_{0}=0.002, \mathrm{f}=6000 \mathrm{r} / \mathrm{min}\right.$, $\varepsilon=0.9)$

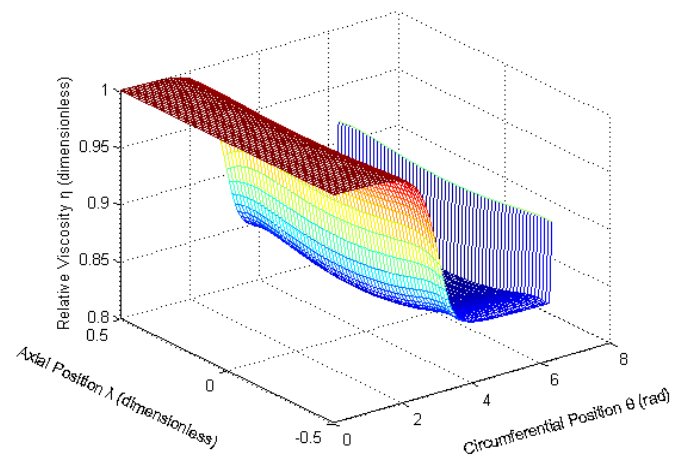

Fig. 8. Viscosity distribution $(\mathrm{h} 0=0.002, \mathrm{f}=6000 \mathrm{r} / \mathrm{min}$, $\varepsilon=0.9$ )

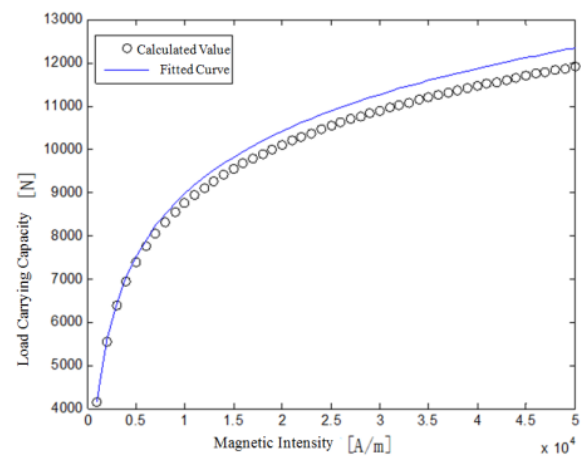

Fig. 9. Influence of magnetic intensity to load carrying capacity 
Clearance h0 is another important parameter that affects bearing performance. By changing clearance from $0.001 \mathrm{~m}$ to $0.003 \mathrm{~m}$ with step $0.0005 \mathrm{~m}$, a set of temperature and pressure curves of median section of bearing are obtained in Fig. 10. It can be seen that clearance dimension has close relationship with temperature and pressure value. The smaller h0 is, the more severely temperature varies and the higher max temperature is, which causes more decrease in fluid viscosity. From (6), fluid pressure is in proportion with the square of $1 / \mathrm{h}_{0}$. Fig. 11 shows pressure of median section under different clearances and Fig. 12 indicates the effect of speed to working temperature.

Rated rotating speed is another concern to design the bearing. Increasing speed means larger Reynolds Number

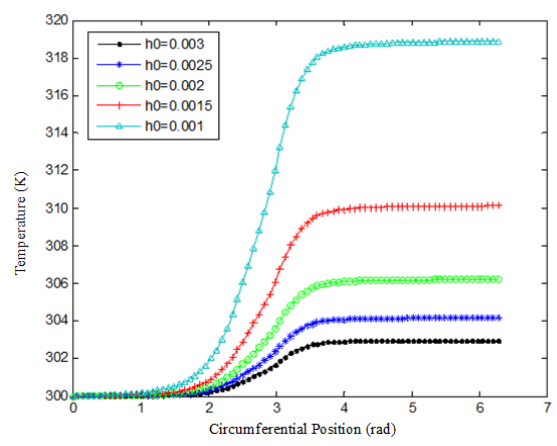

Fig. 10. Influence of clearance to temperature $(\mathrm{f}=6000 \mathrm{r} / \mathrm{min}$, $\varepsilon=0.9$ )

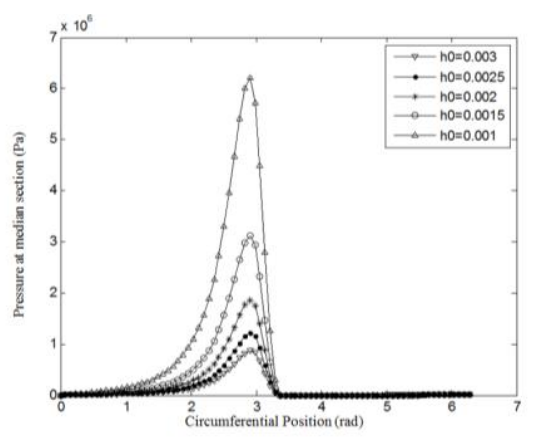

Fig. 11. Influence of clearance to pressure $(\mathrm{f}=6000 \mathrm{r} / \mathrm{min}$, $\varepsilon=0.9$ )

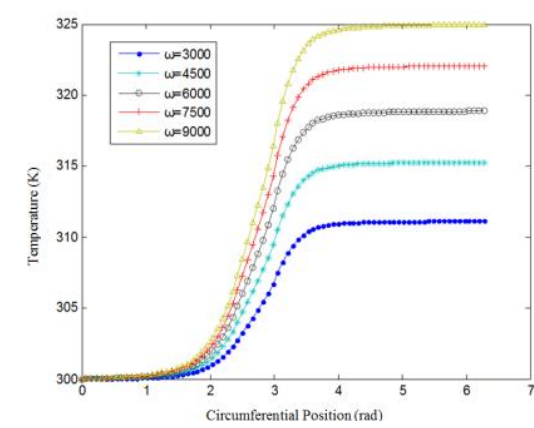

Fig. 12. Influence of speed to temperature $(\varepsilon=0.9)$
(Re), which shall change the main flow state from laminar flow to turbulent flow. Re can be calculated by:

$$
\operatorname{Re}=\frac{\rho \omega r h_{0}}{\eta}
$$

When Re exceeds 10000 , fluid will be almost totally turbulence. Since model used in this paper is based on laminar flow, Re is limited below 2300r/min, i.e. rotating speed below 989.3rad/s (9500r/min).

Despite real fluid viscosity probably larger than what is used in this paper which makes Re less than 2300 even at higher speed, here speed is still set below 9500r/min and this speed is already belong to the high speed category. Changing speed from 3000r/min to $9000 \mathrm{r} / \mathrm{min}$, with step 500r/min, Fig.11 shows the influence of speed to temperature rise. From figure, temperature rise is nearly direct proportion to rotating speed $\omega$. It is mainly because in energy equation (12), fluid velocity is proportion to rotating speed $\omega$, while the source term $\mathrm{S}$ is proportion to square of $\omega$, phenomena discussed above is the combination of these two effects. Fig. 12 compares relationship between load carrying capacity and rotating speed, which shows that under relatively larger clearance, the relationship is almost linear, while it will distort when clearance becomes smaller because of thermal effect.

One thing that we do care about is the difference between ferrofluid and conventional lubricant and whether the former has advantages.

Fig. 13 compares them and the result shows that under same eccentricity, temperature rise in ferrofluid bearing is larger. However, when investigating temperature rise under same load carrying capacity, ferrofluid bearing wins (Fig.14). It means bearings lubricating with ferrofluid can carry heavier load at certain temperature requirement.

\section{Discussion on Bearing Dimension}

Load carrying capacity is a significant performance index to conventional bearing, so is to ferrofluid bearing. Besides, since ferromagnetic property of ferrofluid, magnetic characteristic is also important to the new kind of bearing talked in this paper. As mentioned in chapters above, the two decisive parameters for magnetic bearing can be expressed by:

$$
\left\{\begin{array}{l}
W_{0}=\frac{\eta \omega l r^{3}}{h_{0}{ }^{2}} \\
\alpha=\frac{\mu_{0} X_{m} h_{m 0}{ }^{2}\left(h_{0}{ }^{2}\right)}{\eta \omega l^{2}}
\end{array}\right.
$$




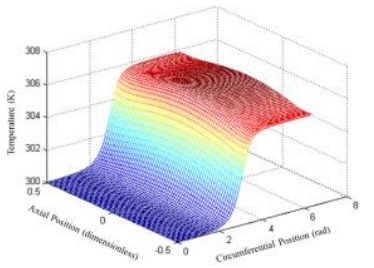

(a). Lubricating with ferrofluid

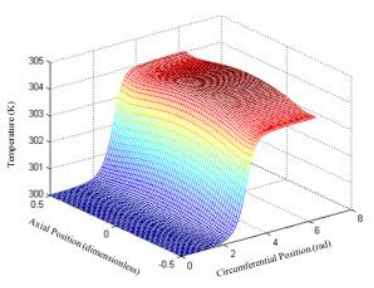

(b). Lubricating with oil
Fig. 13. Lubricating simulation results

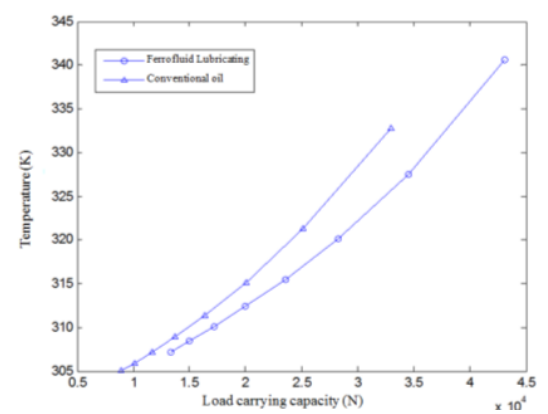

Fig. 14. Working temperature of different lubricant

Where $W_{0}$ is the base value of load capacity and $\alpha$ is magnetic parameter indicating the intensity of external magnetic field.

In terms of increasing load carrying capacity, traditional approach is to extend bearing size and narrow the clearance. Though decrease in clearance can cause rising temperature, but it is ignorable, relatively. This method is also suitable for ferrofluid bearing. In order to make full use of magnetic field, the clearance, however, should be larger with smaller bearing length. It contradicts to the method increasing load capacity. As a result, a compromise should be made according to actual application. If the bearing is going to be used in traction motor on high speed train, the dimension shall be with length $l=0.09 \mathrm{~m}$, radius where film locates $r=0.08 \mathrm{~m}$ and bearing clearance $h_{0}=0.002 \mathrm{~m}$.

\section{Conclusion}

Ferrofluid bearing has better load carrying capacity comparing to conventional one thanks to the additional magnetic effect. By controlling the magnetic parameter, which further changing the lubricant viscosity, this capacity can even be modified to fit different occasions. Through solving modified Reynolds Equation and Energy Equation simultaneously using numerical method, i.e. finite volume method in this paper, several results are obtained which can be concluded as below:

Pressure varies within lubricating area and the peak value locates between $\pi / 2$ and $\pi$. As magnetic effect increased, full-lubricating region extends, which means shrinking of cave region and improving in lubricating characteristics.

Ferrofluid bearings have lower working temperature than conventional lubricated bearings of the same load carrying capacity. In ferrofluid bearings, temperature variation in lubricating area is small. However, since viscosity is sensitive to temperature and changes obviously, thermal effect can't be simply omitted. This paper can be regarded as an extension on the base of Osman's work. Temperature variation, which he omitted, is an important factor to take into consideration since it will bring vibration in fluid viscosity up to approx. $15 \%$. By coupling thermal and hydrodynamic effect, a more reasonable result is obtained as discussed above. Analysis based on temperature distribution is meaningful.

Magnetic effect helps to increase load carrying capacity. As magnetic field becomes stronger, load carrying capacity increases.

Bearing dimension has impact on its properties. When bearing clearance becomes larger, temperature and pressure both deceases.

In this study, radical heat transmission isn't considered. Another influence which is caused by eddy current during rotating when metal shaft cuts magnetic flux is not considered, either. This work has been studied by H. Yang and S. Jin. Commercial software such as ANSYS and FLUENT may be helpful when doing the job. Considering special characteristics of ferrofluid, theoretical derivation and free hand work will still be necessary.

\section{Acknowledgements}

This project is supported by the National Natural Science Foundation of China (No. 51105331) and the National High Technology Research and Development Program of China (863 Program) (No.2011AA11A101).

\section{References}

[1] Ronald E. Rosensweig, "Magnetic fluids", Annual Reviews. Fluid Mech. vol.19, pp.431-461, January 1987.

[2] S. Odenbach, "Ferrofluids-magnetically controlled suspension", Colloids and Surfaces A: Physicochem, vol. 217, no.1, pp.171-178, April 2003.

[3] J. M. Guldbakke, J. Hesselbach, "Development of bearings and a damper based on magnetically controllable fluids", Journal of Phiysics: Condensed Matter, vol.18, no.38, pp.2959-2972, September 2006.

[4] W. Ochonski, "Sliding bearings lubricated with magnetic fluids", Industrial Lubrication and Tribology, vol.59, no.6, pp.252-265, 2007.

[5] Nicolae Tipei, "Theory of Lubrication With Ferrofluids: Application to Short Bearings," Transactions of the ASME, vol. 104, no.5, pp. 510-515, October 1982. 
[6] H.S. Chang, C.Q. Chi, P.Z. Zhao, "A theoretical and experimental study of ferrofluid lubricated four-pocket journal bearings", Journal of Magnetism and Magnetic Materials, vol. 65, no. 2-3, pp. 372-374, March 1987.

[7] T.A. Osman, G.S. Nada, Z.S. Safar, "Static and dynamic characteristics of magnetized journal bearings lubricated with ferrofluid", Tribology International, vol. 34, no. 6, pp. 369380, June 2001.

[8] H. Yang, J.E. Ma, X.Y. Huang, Y.T. Fang, "Eddy Current Loss Analysis of Sliding Bearing for Active Excitation Magnetic Fluid Lubrication”, Small \& Special Electrical Machines, vol. 2011, no. 7, pp. 43-45, July 2011.

[9] S. Jin, J.E. Ma, X.L. Li, X. Yin, Y.T. Fang, "ANSYS Simulation and Performance Analysis Under Eccentricity for Magnetic Fluid Bearings", Bearing, vol. 2012, no. 2, pp. 29-32, February 2012.

[10] P. Kuzhir, "Free boundary of lubricant film in ferrofluid journal bearings", Tribology, vol.41, no.4, pp.256-268, April 2008.

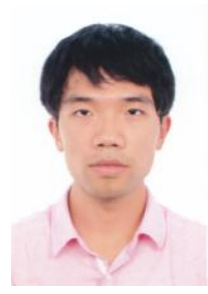

Xin Yin received B.S degree in electrical engineering from Zhejiang University. He is currently studying in Zhejiang University for the Ph.D. degree. His main research interests include PM machines and drives and mechatronics machines such as ferrofluid bearing.

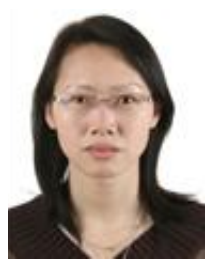

Jien Ma received the Ph.D. degree in mechatronics from Zhejiang University. Currently, she is a postdoctor with the College of Electrical Engineering, Zhejiang University, where she is working on electrical machines and drives. Her research interests include the PM machines and drives for traction applications, mechatronics machines such as magneto fluid bearing.

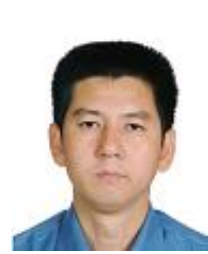

Youtong Fang received the Ph.D. degree in electrical engineering from Hebei University of Technology. His research interests include high-speed railway technology, new energy utilization, permanent magnet motor and magnetic levitation technology. $\mathrm{He}$ is also Member of Overall Experts Group for China Autonomous Innovation Joint Action Plan of High Speed Train and Executive Directors of the Electric Reliability Council of China Electro-technical Society.

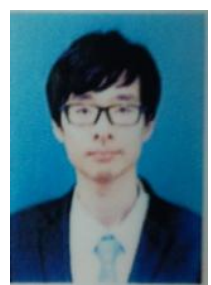

Shuai Jin received Master degree in electrical engineering from Zhejiang University. His research interests include PM machines and mechatronics machines such as ferrofluid bearing. 